\title{
双动人工半嚗关节假体的设计及应用*
}

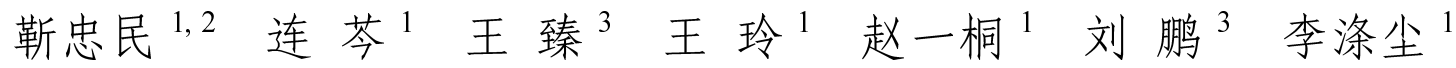

(1. 西安交通大学机械制造系统工程国家重点试验室 西安 710049;

2. 利兹大学医学生物研究所 利兹 LS2 9JT 英国;

3. 第四军医大学西京医院 西安 710032)

\begin{abstract}
摘要: 建立一种双动人工半膝关节假体的设计方法, 用于年轻病人因恶性骨肿瘤造成的股骨远端大段骨缺损的保肢手术治疗。 借助于有限元分析与快速成形技术, 研究并快速而有效地解决假体结构设计优化、生物力学分析、稳定性评估等技术难点, 形成一种以定制化仿生大范围股骨髅部、滑动轴套系统、韧带附丽孔道为特点的附丽型定制化双动半膝关节假体。临床应用 证明置换术后假体与周围组织匹配性良好, 患者在完成深屈曲运动时可观察到假体的双滑动过程。该假体的研究为单侧膝关 节损伤患者, 特别是青少年患者术后骨生长的生物学重建及后期关节功能的良好恢复提供了有效的治疗方法。该研究也从功 能化设计、性能分析与临床功能评估等方面为人工关节的研制与发展提出新的思路与方法。
\end{abstract}

关键词: 半膝关节人工假体 双动策略 功能化设计 生物力学

中图分类号: TB17

\section{Custom-made Bipolar Hemi-knee Prosthesis Design and Clinical Application}

\author{
JIN Zhongmin ${ }^{1,2}$ LIAN Qin $^{1}$ WANG Zhen ${ }^{3}$ WANG Ling ${ }^{1}$ \\ ZHAO Yitong ${ }^{1}$ LIU Peng $^{3} \quad$ LI Dichen $^{1}$
}

(1. State Key Laboratory of Mechanical System Engineering, Xi'an Jiaotong University, Xi'an 710049;

2. Institute of Medical and Biological Engineering, University of Leeds, Leeds LS2 9JT, UK;

3. Xijing Hospital, Fourth Military Medical University, Xi'an 710032)

\begin{abstract}
A bipolar customised hemi-knee prosthesis is designed to treat maligament bone tumor on the femoral side of a young patient. Design considerations included the close match with the patients anatomy, stress optimisation of the prosthetic components, close conformity between the contacting surfaces of the prosthesis and the tibial cartilage and stability as well as reducing the development time and costs. Computational analysis based on the finite element method is employed to optimise the designs and provide pre-clinical evaluation. The optimised prosthesis was fabricated via rapid manufacture technique and tested in-vitro to evaluate its effectiveness. Clinical application of the prosthesis shows improved functional outcomes. It not only proposes a new concept of bipolar hemi-knee prosthesis, but also provides a general approach for functional design, computational analysis, rapid manufacturing and clinical application of customised prostheses.
\end{abstract}

Key words: Hemi-knee joint prosthesis Bipolar strategy Functional design Biomechanics

\section{0 前言}

外科重建技术及现代医学影像诊断技术的发 展进步, 使得临床上 $90 \%$ 的恶性骨肿瘤患者可以通 过保肢重建手术获得治疗。不过, 由于全膝关节置

* 国家自然科学基金资助项目(51075320, 50628505)。20121205 收到初稿, 20130220 收到修改稿
换术需要切除患者膝关节的股骨远端、半月板和胫 骨近端，导致术后患者股骨和胫骨的关节骨骺的严 重破坏，对于单侧关节损伤的青少年患者，甚至出 现术后双侧下肢的不等长、下腰部疼痛、代偿性脊 柱侧弯及步态异常等问题。因此全膝关节置换术并 不是最佳的保肢治疗方法 ${ }^{[1-2]}$ 。

利用患者诊断影像数据和计算机辅助设计, 以 及目前越来越被重视的快速成形技术(如光固化成 
形、激光烧结、三维打印等), 定制化假体正在快速 发展, 并开始应用于临床 ${ }^{[3-8]}$ 。通过针对患者肿瘤切 除后进行的患侧大段骨缺损, “量身定做”定制化半 膝关节假体进行手术置换, 很有希望成为新的保肢 治疗方法 ${ }^{[3-5]}$ 。而且, 该方法也为治疗膝关节退化性 关节疾病提供了新的临床治疗手段。

目前国内外在研究定制化关节假体的主要方 法是首先利用反求工程技术处理图像数据, 建立患 者膝关节三维模型; 再在该模型基础上设计出可以 与患者关节面完全匹配的假体, 最终实现降低术后 假体松动和脱位的风险率, 并减少关节面磨损程度 的目的。定制化假体设计流程主要以 $\mathrm{CAD} / \mathrm{CAM}$ 为 核心技术，包括有图像数据处理、三维模型重建、 匹配假体设计和 CAM 等系列单元技术 ${ }^{[8-12]}$ 。图像数 据处理是为了获取患者膝关节诊断影像数据; 三维 模型重建是为了建立了患者患处膝关节的三维实体 模型; 匹配假体设计是利用 CAD 软件的匹配设计 功能来实现获得定制化的人工关节假体模型; CAM 是利用以快速成形技术为代表的计算机辅助制造技 术加工制造出高精度的定制化设计假体。CAD 技术 和有限元分析法的结合可以在完成假体设计中模拟 优化设计参数, 研究证明是一种可信有效的假体设 计和临床前测试方法 ${ }^{[13-15]}$ 。

HARRYSSON 等 ${ }^{[16]}$ 利用有限元分析法研究评 价定制化股骨假体置换的性能, 计算分析结果发现 定制化设计的假体相较于非定制化的在骨与假体界 面上有着更加均匀的应力分布, 有效地降低了假体 松动和脱位的风险率。JUN 等 ${ }^{[17]}$ 开发设计出一套定 制化髋关节股骨假体的软件系统, 能够有效地设计 包括股骨解剖轴线、股骨头半径、股骨柄长度、假 体外翻角等个性化参数, 通过临床试验验证, 证明 该系统可以有效地完成假体设计, 系统运行良好。 HEEVER 等 ${ }^{[18]}$ 利用健康膝关节几何形态数据定制 化设计出膝关节髅置换假体, 并采用有限元分析假 体置换后骨与假体界面间应力分布情况时, 发现定 制化假体置换后骨与假体应力分布相较于传统的固 定置换假体和移动支撑假体更加均匀, 而且应力大 小也低于二者, 减低了假体磨损、松动和脱位的风 险率。

现有定制化人工半膝关节假体并未在临床上 获得广泛应用。主要是由于现有半膝关节假体置换 后近期临床效果虽然较好, 但长远期效果不好。主 要表现为假体脱位、稳定性差, 假体植入后易出现 对侧膝关节软骨被严重磨损的情况。普遍认为这是 由于假体的材料选择与结构设计不合理, 导致的应 力集中、应力屏蔽和关节面不匹配 ${ }^{[19-20]}$ 。因此, 现
有半膝关节假体与对侧关节面匹配性结构的设计方 法仍存在较大问题。

西安交通大学机械工程学院与第四军医大学 西京医院凭借各自在设计与临床上的优势, 依托快 速成形技术, 2003 年联合研制出了第一代定制化人 工半膝关节假体 ${ }^{[21]}$ (图 1)。该假体能够满足膝关节力 学强度和关节匹配性要求。经临床试验和随访发现, 定制化假体明显改善了膝关节功能 ${ }^{[21-22]}$ 。随后, 借 鉴人工髋关节置换术中 Christian 人工假体上的股骨 头双动策略 ${ }^{[23]}$ 又相继推出的第二代定制化半膝关 节假体增加双动和附丽设计 ${ }^{[24]}$ 。

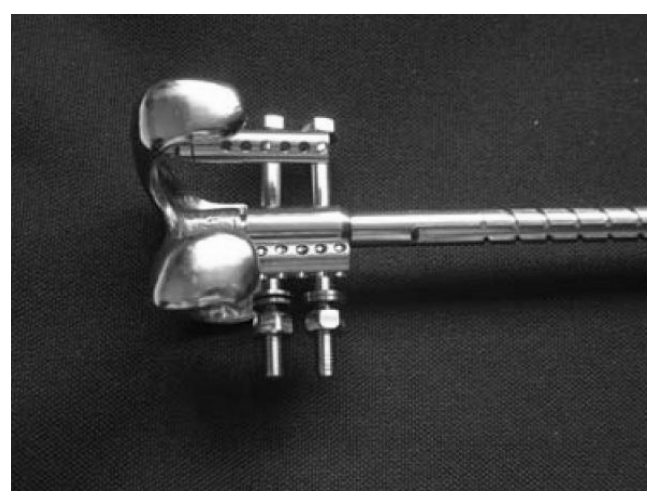

图 1 定制化人工半膝关节金属假体

本文以一例恶性骨肿瘤造成的股骨远端大段 骨缺损的保肢手术治疗为例, 从假体定制化结构设 计、摩擦学性能分析与临床前稳定性评估、临床应 用效果等方面, 介绍附丽型定制化双动半膝关节假 体的设计与应用的方法, 并分析半膝关节的定制化 与双滑动结构对膝关节生物力学环境的重建与运动 稳定性等方面的影响, 讨论双动半膝关节假体在设 计制造、分析评估、临床应用上等面临的难点与挑 战, 及其最新研究进展。为发展和研制新型人工植 入物探索新的思路和制造技术。

\section{1 临床问题与假体设计方法}

\section{1 临床问题与设计要求}

儿童患者, 女性, 1 岁时右侧股骨发现巨大恶 性神经鞘瘤。确定的治疗方案为切除股骨肿瘤段并 利用大段同种异体骨移植的保肢治疗方法, 成功地 保住了患者的肢体。但是 4 岁时发现由于异体骨植 入物的股胫骨关节面的不匹配, 使得早期植入的异 体肱骨髁部发生骨折。经过第四军医大学与西安交 通大学研究人员共同研究, 提出采用附丽型定制化 双动半膝关节假体进行置换手术。设计要求如下:

(1) 半膝关节假体应能与周围组织进行良好的匹配, 尽可能避免和降低对对侧胫骨或半月板的磨损, 延 
缓假体的翻修时间; (2) 假体置换后膝关节应能保证 大部分屈曲旋转运动; (3) 假体置换后应能保证关节 运动的稳定性, 要求假体上构建人工韧带附丽系统, 减少关节软组织损伤、假体脱位和翻修风险。

\section{2 假体滑动设计原理}

为了使得假体置换后膝关节能完成屈曲旋转 运动, 并减少摩擦磨损, 把人工半膝关节假体运动 由原先直接跟对侧软骨直接接触与滑动的过程, 改 变为金属对金属和金属对软骨两部分。即根据人体 膝关节运动过程中股骨头与对侧胫骨的运动轨迹特 点, 在半膝关节假体上安装滑动轴套机构, 置换该 假体后会将膝关节运动分成两个阶段：第一阶段为 膝关节屈曲角度 $0^{\circ} \sim 60^{\circ}$, 即从图 $2 \mathrm{a}$ 状态到图 $2 \mathrm{~b}$ 状态, 通常为人体正常步态角度, 此时假体相对于 胫骨平台进行前后移动的幅度较小; 此时, 以金属 对金属滑动为主。第二阶段是指当膝关节屈曲角度 超过 $60^{\circ}$, 即见图 $2 \mathrm{~b}$ 状态到图 $2 \mathrm{c}$ 状态, 如蹲、趾 等深度屈曲状态, 假体开始相对于胫骨平台有较大 的前后移动位移。此时, 以金属对软骨滑动为主。 这样, 通过在假体上设计滑动机构降低假体与对侧 胫骨软骨运动摩擦频率和接触位移, 不仅降低了假 体对胫骨平台软骨的磨损, 亦可保证患者胫骨的正 常生长。

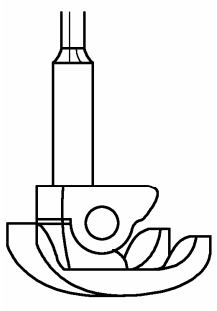

(a) $0^{\circ}$

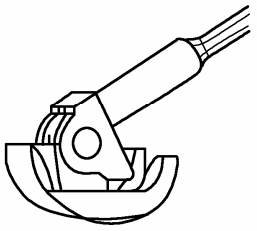

(b) $60^{\circ}$

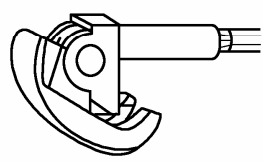

(c) $90^{\circ}$
图 2 膝关节假体关键姿态角度图

\section{3 双动人工半膝关节假体设计方案}

根据设计要求, 将双动定制化膝关节假体分股 骨假体接合部、股骨髁仿生部、滑动轴套系统三个 部分进行设计。根据人体韧带的解剖学位置, 在股 骨髁仿生部上, 即在股骨端内外侧副韧带附着点和 股骨髁端前后交叉韧带附着点设计韧带附着孔道。

为了获得半膝关节假体的定制化股骨髁远端表 面, 确定以基于断层扫描技术(Computed tomography, CT)扫描图像数据建立患者病患处膝关节的三维实体 模型, 即利用 Mimics V 8.0, Geomagic Studio V 8.0, Pro/E V3.0, ANSYS V 10.0 进行计算机辅助设计。以 膝关节 $\mathrm{CT}$ 断层图像数据(女性儿童患者, 年龄 5 岁, 体重 $35 \mathrm{~kg}$ ，见第 1.1 节)为基础, 构建三维膝关节三 维模型, 半膝关节假体模型、膝关节有限元模型。

\section{4 假体置换后膝关节生物力学分析方案}

本研究中假体材料为钛合金材料, 其抗拉强度 $\sigma_{\mathrm{b}}=860 \mathrm{MPa}$, 屈曲强度 $\sigma_{u}=378 \mathrm{MPa}$ 。假体的可 靠性和疲劳分析采用 Goodman 疲劳理论公式 ${ }^{[25]}$ 。 在假体有限元计算分析中, 最小等效应力一般接近 为零, 即 $\sigma_{\text {min }} \approx 0$, 因此可得到分析公式

$$
\left(\frac{1}{\sigma_{\mathrm{b}}}+\frac{1}{\sigma_{u}}\right) \sigma_{\max }=\frac{2}{N}
$$

式中 $\sigma_{\text {max }}$ 一最大等效应力

$N$ 一安全系数

由于 $1 / \sigma_{\mathrm{b}}+1 / \sigma_{u}$ 为常数, 那么假体的安全系数 $N$ 与最大等效应力 $\sigma_{\max }$ 成反比, 最大等效应力越大, 安全系数越低。因此, 本研究中, 采用假体和股骨 的所受的最大应力作为评估假体设计参数的合理性 的分析指标。

由于定制化表面对生物力学环境的影响是通 过假体股骨髅仿生部的设计来满足，因此假体的主 要设计参数研究应该是针对股骨假体结合部参数进 行的优化分析, 即股骨假体结合部的三个设计参数: 髓内柄截面、髓内柄长度、股骨假体结合部的外翻 角。采用正交试验法, 利用有限元法计算各设计参 数变化时, 假体和膝关节股骨上范式应力的变化情 况, 研究三个设计参数对膝关节力学环境重建的联 合作用。

设定外翻角、髓内柄长度、髓内柄截面形状为 三个因素, 每个因素建立 5 个水平值, 具体如下: 外翻角 5 个水平值分别为 $6.0^{\circ} 、 6.2^{\circ} 、 6.4^{\circ}$ 、 $6.6^{\circ}$ 和 $6.8^{\circ}$; 髓内柄长度分别是 $50 \mathrm{~mm} 、 60 \mathrm{~mm}$ 、 $70 \mathrm{~mm} 、 80 \mathrm{~mm}$ 和 $90 \mathrm{~mm}$; 髓内柄截面形状为 4 个 水平值, 分别为垂直纺锤形截面、平行纺锤形截面、 圆形截面和六边形截面, 其中垂直是指纺锤形长轴 垂直于旋转中轴线, 平行而反之。设计参数优化分 析的因素水平表见表 1 。

表 1 参数优化分析因数水平表

\begin{tabular}{cccc}
\hline \multirow{2}{*}{ 水平 } & \multicolumn{4}{c}{ 因素 } \\
\cline { 2 - 4 } & 外翻角 $/\left(^{\circ}\right)$ & 柄长度 $/ \mathrm{mm}$ & 截面形状 \\
\hline 1 & 6.0 & 50 & 垂直纺锤形 \\
2 & 6.2 & 60 & 平行纺锤形 \\
3 & 6.4 & 70 & 圆形 \\
4 & 6.6 & 80 & 六边形 \\
5 & 6.8 & 90 & - \\
\hline
\end{tabular}

模拟行走状态下，分析假体置换后的单膝极限 受力情况, 即分析假体置换对临床力学指标的影响, 研究假体结构特征对膝关节生物力学环境的重建作 用。力学环境分析假设如下: (1) 由于较高的应力峰 
值处易发生假体损坏, 降低假体的使用寿命, 所以 应避免假体置换后出现高应力峰值; (2)由于应力屏 蔽的出现, 会使置换区的宿主骨因所受应力不足, 产生骨质疏松、皮质骨变薄等问题, 造成患者二次 伤害，所以应避免置换后出现应力屏蔽。

设定股-胫关节压缩力在行走负重相最大负荷 为体重的 4 倍 ${ }^{[26]}$ 。由于患者体重 $35 \mathrm{~kg}$, 所以在股 骨头球心加载 $1372 \mathrm{~N}(4$ 倍患者体重)的压力, 约束 和加载条件见图 3 。模拟人体真实膝关节负重情况, 将图 3 中加载力的角度设定为股骨机械轴与股骨髁 上轴线的垂直线之间的夹角, 夹角值通过 Pro/E 软 件测量获得。通过 ANSYS 软件与 Pro/E 软件的无 缝连接, 实现 CAD 到 CAE 的文件无损传输。ANSYS 软件下通过中的 GLUE 命令进行股骨与假体的布尔 连接。

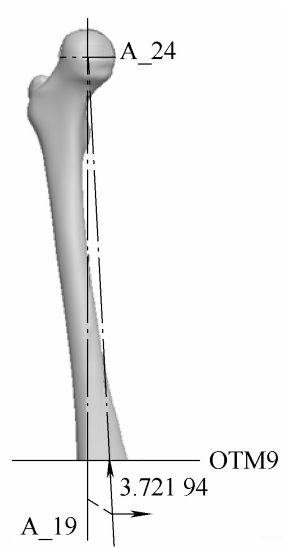

(a) 加载力角度

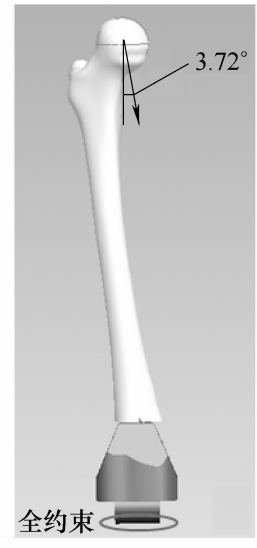

(b) 边界条件

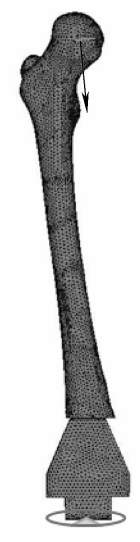

(c) 有限元模型
图 3 设计参数有限元模型与边界条件

设置骨与假体的网格单元为 SOLID92。假体由 钛合金(Ti-6Al-4V)完成, 材料属性见表 2。

表 2 组织材料属性

\begin{tabular}{ccc}
\hline \multirow{2}{*}{ 材料列表 } & \multicolumn{2}{c}{ 材料属性 } \\
\cline { 2 - 3 } & 弹性模量/MPa & 泊松比 \\
\hline 骨 & 11500 & 0.30 \\
软骨 & 15 & 0.30 \\
半月板 & 59 & 0.45 \\
钢合金 & 110000 & 0.49 \\
\hline
\end{tabular}

\section{5 假体滑动面摩擦学性能分析方案}

软骨的磨损和损坏直接由摩擦切应力 $\tau$ 决 定 $^{[27]}$, 即

$$
\tau=\sigma \mu_{e f f}
$$

式中

$\tau$ 一摩擦切应力

$\sigma$ 一接触应力

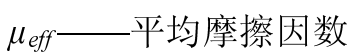

因此, 滑移接触面间接触应力的分析可作为膝 关节假体对对侧软骨磨损影响的定量评估方法。本 文以自然膝关节和定制化半膝关节假体为对照组, 对比研究定制化双动半膝关节假体植入体内后对膝 关节对侧关节面的接触应力情况, 并对比分析来半 膝关节假体在减轻膝关节对侧关节面摩擦和磨损方 面的可能性。

设置骨与软骨组织网格单元为 SOLID92。接触 对(CONTACT PAIR) 确定为面-面接触, 目标面为 TARGE170 单元，接触面为非线性 CONTA174 单元。 骨与软骨为 GLUE 粘结, 股骨髁软骨、内外侧半月 板和胫骨内外侧软骨六个相应的接触面通过接触对 设置成为接触分析所用的接触对; 股骨髁两侧垂直 平面设置为仅有垂直向下运动的自由度; 内外侧半 月板靠近胫骨平台中央处的两端因粘结在胫骨内外 侧软骨上, 故将其两端进行固定全约束; 胫骨的下 端进行全约束。自然膝关节的有限元模型中, 设定 典型网格单元长度为 $2 \mathrm{~mm}$, 股骨和胫骨模型共有 260162 个网格单元, 接触模型(股骨软骨、半月板 和胫骨软骨)共有 108801 个网格单元, 整个有限元 模型共有 368963 的网格单元。计算三组关节接触 面关节屈曲角度选择在 $0^{\circ} 、 30^{\circ}$ 、 $60^{\circ}$ 和 $90^{\circ}$ 的瞬间接触应力。

接触应力分析中, 一般将人工假体设定为线弹 性材料, 将生物材料, 即骨、软骨、半月板等设定 为多孔弹性材料。但在本研究分析中将有限元模型 (图 4)简化为线弹性模型, 其材料属性见表 2 。

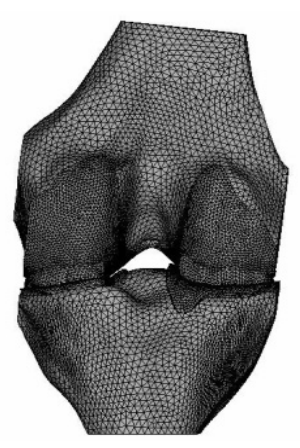

(a) 自然膝关节

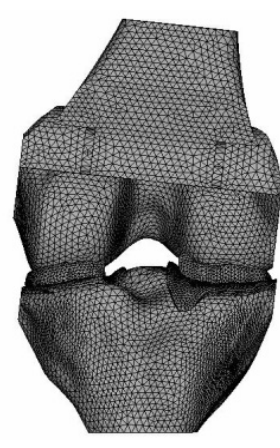

(b) 双动半膝假体关节
图 4 接触力学分析计算的有限元模型

\section{2 稳定性试验和临床试验方案}

\section{1 体外稳定性试验方案}

为了验证韧带附丽系统的稳定性, 分别模拟假 体植入后假体关节屈曲运动下和在特殊极限(胫骨 前移和后移极限状态)下㓞带的受力情况。利用光固 化快速成型机(SPS600B, 陕西恒通智能机器有限公 
司)制造双动半膝关节假体树脂模型和胫骨近端树 脂模型。然后将两条 LARS 韧带分别穿过附丽孔道, 韧带两头固定于胫骨平台上。

将外叉韧带安装在假体弯曲度为 $0^{\circ}$ 处, 内叉 韧带安装在假体弯曲度为 $105^{\circ}$ 处。模拟膝关节假 体在不同屈曲角度下旋转, 在 $60^{\circ}$ 和 $135^{\circ}$ 之间每 隔 $15^{\circ}$ 测量所需韧带长度, 并与初始韧带长度对比 计算其长度差值。模拟膝关节植入后在胫骨前移和 后移的极限状态下, 测量所需韧带长度, 并与初始 㓞带长度对比计算其差值, 根据式(3)计算韧带力

$$
F=E S_{0} \Delta \varepsilon
$$

式中 $E$ 一一弹性模量

$S_{0}$ ——初始截面积

$\Delta \varepsilon$ 一一伸长率

\section{2 临床试验方案}

将患者置于仰卧位, 以右侧大腿内侧原有手术 切口为基准切口, 依次用手术刀切开皮肤和皮下组 织后, 分离开股直肌和股侧肌的缝隙。剥离移植骨 和髌骨粘结处, 分离移植骨与胫骨平台。在股骨髁 远端截断骨干，摘除异体骨。扩孔股骨髓腔，插入 假体髓内柄。假体安装完成后再用 LARS 人工㓞带 将假体与胫骨连接, 并在关节内侧进行打结紧固。 确定膝关节的活动度良好后, 冲洗伤口, 闭合切口。 将患者送返病房。手术后 $\mathrm{X}$ 光片和短期随访分析假 体治疗效果。

\section{3 结果与讨论}

\section{1 患者个体病变膝关节模型}

利用计算机 $X$ 射线断层扫描技术扫描图像数据 构建的患者病患处膝关节模型见图 5。具有生理状 态下的完整匹配关系的股胫模型将为后续建立半膝 关节假体的曲面结构和膝关节力学环境的有限元分 析提供条件。

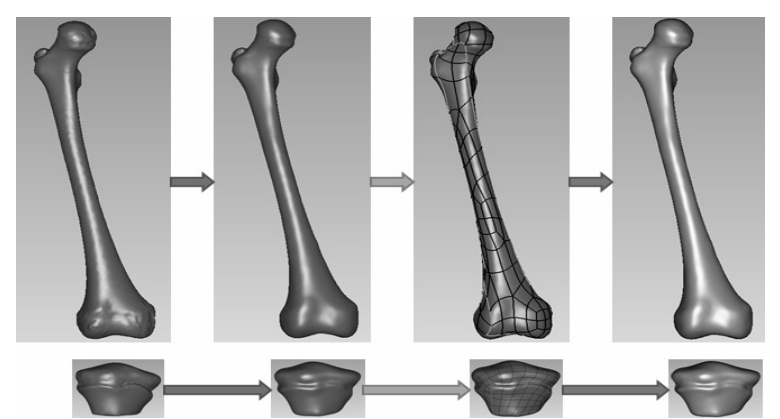

图 5 患者膝关节股胫模型

\section{2 附丽型定制化双动半膝关节假体}

半膝关节假体设计模型主要包括股骨假体、轴 套系统和股骨髁体三部分, 见图 6。股骨髁体上髁 面曲面来自患者膝关节股骨头, 能够保证假体与胫 骨平台及髌骨关节面接触面形成良好匹配关系。

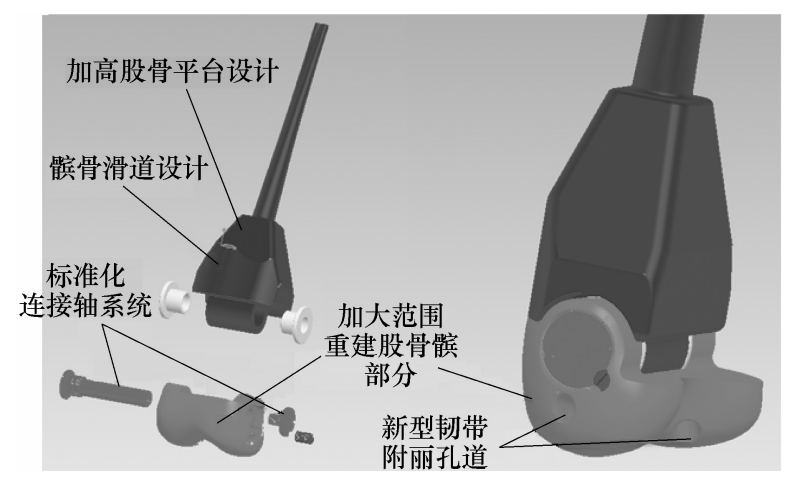

图 6 附丽型双动半膝关节假体结构设计模型

针对假体运动的稳定性问题, 在股骨髁体处, 在人体韧带的解剖学位置建立韧带附丽孔道, 即在 股骨端内外侧副韧带附着点和股骨髁端前后交叉韧 带附着点建立韧带附着孔道。利用 2 条 LARS 人工 韧带来替代 4 条主要韧带(内外侧副韧带和前后交 叉㓞带), 见图 7。

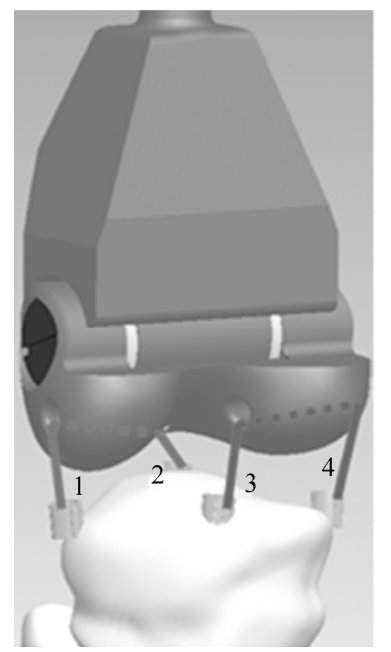

图 7 附丽型双动假体㓞带附丽图

\section{3 假体置换后生物力学分析}

假体置换后，膝关节处假体和股骨的典型应力 分布情况见图 8。正交试验各组分析结果见表 3。

正交试验直观分析计算得出的均值用来判 断某因素中哪种水平对于参照来说是最优的水平, 而极差值用来判断哪种因素对试验指标的影响 程度。利用正交试验计算结果, 分别计算各因素下 假体最大应力和股骨最大应力的均值与极值, 列入 表 4、5。 


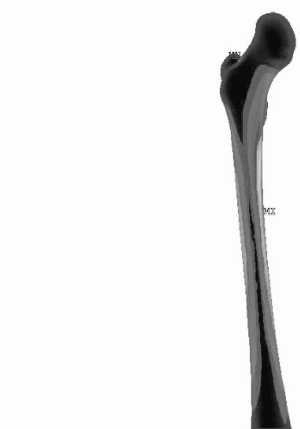

$\begin{array}{lllll}0.056 & 12.359 & 24.661 & 36.964 & 49.267\end{array}$

(a) 股骨计算分析

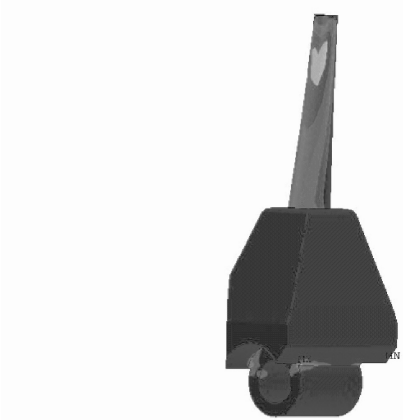

0.005

\subsection{2}

图 8 试验 $1(\mathrm{~A} 1 \mathrm{~B} 1 \mathrm{C} 1$ 组合)下股骨和假体上范式应力 分布云图(MPa)

表 3 参数优化正交试验计算结果

\begin{tabular}{|c|c|c|c|c|c|}
\hline \multirow[b]{2}{*}{ 试验组 } & \multicolumn{3}{|c|}{ 因素 } & \multicolumn{2}{|c|}{ 应力分析值/MPa } \\
\hline & $\begin{array}{l}\mathrm{A}(\text { 外 } \\
\text { 翻角) }\end{array}$ & $\begin{array}{l}\text { B(髓内 } \\
\text { 柄长度) }\end{array}$ & $\begin{array}{l}\mathrm{C} \text { (髓内 } \\
\text { 柄形状) }\end{array}$ & 假体 & 股骨 \\
\hline 1 & A 1 & B1 & $\mathrm{C} 1$ & 129.321 & 55.419 \\
\hline 2 & A 1 & B2 & $\mathrm{C} 2$ & 171.565 & 124.959 \\
\hline 3 & A1 & B3 & $\mathrm{C} 3$ & 133.327 & 70.998 \\
\hline 4 & A1 & B4 & $\mathrm{C} 4$ & 177.288 & 85.472 \\
\hline 5 & $\mathrm{~A} 2$ & B1 & $\mathrm{C} 2$ & 155.065 & 114.640 \\
\hline 6 & $\mathrm{~A} 2$ & B2 & $\mathrm{C} 3$ & 165.941 & 59.688 \\
\hline 7 & A2 & B3 & $\mathrm{C} 4$ & 142.321 & 65.132 \\
\hline 8 & $\mathrm{~A} 2$ & B5 & $\mathrm{C} 1$ & 167.645 & 88.102 \\
\hline 9 & A3 & B1 & $\mathrm{C} 3$ & 154.910 & 66.817 \\
\hline 10 & A3 & B2 & $\mathrm{C} 4$ & 160.792 & 58.804 \\
\hline 11 & A3 & B4 & $\mathrm{C} 1$ & 157.971 & 60.949 \\
\hline 12 & A3 & B5 & $\mathrm{C} 2$ & 179.610 & 120.685 \\
\hline 13 & A4 & B1 & $\mathrm{C} 4$ & 172.063 & 74.757 \\
\hline 14 & A4 & B3 & $\mathrm{C} 1$ & 163.251 & 54.548 \\
\hline 15 & A4 & B4 & $\mathrm{C} 2$ & 170.924 & 140.009 \\
\hline 16 & A4 & B5 & $\mathrm{C} 3$ & 207.168 & 76.384 \\
\hline 17 & A5 & B2 & $\mathrm{C} 1$ & 211.163 & 75.702 \\
\hline 18 & A5 & B3 & $\mathrm{C} 2$ & 150.657 & 115.018 \\
\hline 19 & A5 & B4 & $\mathrm{C} 3$ & 147.793 & 76.020 \\
\hline 20 & A5 & B5 & $\mathrm{C} 4$ & 208.569 & 74.237 \\
\hline
\end{tabular}

表 4 各因素下假体应力均值和极差值

$\mathrm{MPa}$

\begin{tabular}{cccc}
\hline \multirow{2}{*}{ 水平组 } & \multicolumn{3}{c}{ 因素 } \\
\cline { 2 - 4 } & 外翻角 & 髓内柄长度 & 髓内柄形状 \\
\hline 1 & 122.300 & 122.272 & 165.870 \\
2 & 126.194 & 141.892 & 165.964 \\
3 & 120.423 & 117.911 & 167.828 \\
4 & 142.681 & 130.795 & 172.207 \\
5 & 143.636 & 152.598 & - \\
\hline 极差值 & 23.213 & 34.687 & 6.337 \\
\hline
\end{tabular}

表 5 各因素下股骨应力均值和极差值 $\mathrm{MPa}$

\begin{tabular}{cccc}
\hline \multirow{2}{*}{ 水平组 } & \multicolumn{3}{c}{ 因素 } \\
\cline { 2 - 4 } & 外翻角 & 髓内柄长度 & 髓内柄形状 \\
\hline 1 & 67.370 & 62.327 & 66.944 \\
2 & 65.512 & 63.831 & 123.062 \\
3 & 61.451 & 61.139 & 69.981 \\
4 & 69.140 & 72.490 & 71.680 \\
5 & 68.195 & 71.882 & - \\
\hline 极差值 & 7.689 & 11.351 & 56.118 \\
\hline
\end{tabular}

对比各因素和水平下的假体最大应力值(表 4), 确定假体最优设计参数组合为 $\mathrm{A} 3 \mathrm{~B} 3 \mathrm{C} 1$, 即外翻角 为 $6.4^{\circ}$ 、髓内柄长度为 $70 \mathrm{~mm}$ 及髓内柄截面为垂 直纺锤形结构。从假体应力极值的对比分析, 确定 髓内柄长度对假体应力指标的影响最大, 其次是外 翻角, 最后是髓内柄截面形状。

对比各因素和水平下的股骨最大应力值(表 5), 假体最优设计参数组合为 $\mathrm{A} 3 \mathrm{~B} 3 \mathrm{C} 1$, 即外翻角为 $6.4^{\circ}$ 、髓内柄长度为 $70 \mathrm{~mm}$ 及髓内柄截面为垂直 纺锤形结构, 与表 4 的结果一致。但对比股骨应力 极值分析发现，股骨柄截面对股骨应力指标的影响 是最大, 其次是股骨柄长度, 最后是外翻角。

综上所述, 确定 $\mathrm{A} 3 \mathrm{~B} 3 \mathrm{C} 1$ 为假体设计参数的优 化组合, 即外翻角为 $6.4^{\circ}$ 、髓内柄长度为 $70 \mathrm{~mm}$, 髓内柄截面为垂直纺锤形结构。

\section{4 假体滑动面接触力学分析}

膝关节屈曲典型状态相时各组接触应力结果 见表 6、7。其中, 定制化双动半膝假体关节的关节 面最大接触应力除了在初始典型状态相 $($ 膝关节屈 曲角度为 $0^{\circ}$ )外, 均小于对照组, 且半月板处接触 应力最大值为 $7.720 \mathrm{MPa}$; 而半膝关节假体组半月 板接触应力最大值为 $9.327 \mathrm{MPa}$, 超出自然膝关节 半月板接触应力值较多。对侧胫骨平台上软骨应力 的变化与半月板出现了类似的情况。

表 6 膝关节屈曲典型状态相半月板最大接触应力 $\mathrm{MPa}$

\begin{tabular}{lcccc}
\hline \multirow{2}{*}{ 计算模型 } & \multicolumn{4}{c}{ 屈曲角度 $\left(^{\circ}\right)$} \\
\cline { 2 - 5 } & 0 & 30 & 60 & 90 \\
\hline 自然膝关节 & 7.505 & 7.868 & 5.900 & 7.973 \\
双动半膝关节 & 7.720 & 7.140 & 4.999 & 6.521 \\
半膝关节 & 7.735 & 9.103 & 7.916 & 9.327 \\
\hline
\end{tabular}


表 7 嚗关节屈曲典型状态相胫骨软骨最大接触应力 $\mathrm{MPa}$

\begin{tabular}{lcccc}
\hline \multirow{2}{*}{ 计算模型 } & \multicolumn{4}{c}{ 屈曲角度 } \\
\cline { 2 - 5 } & $0^{\circ}$ & $30^{\circ}$ & $60^{\circ}$ & $90^{\circ}$ \\
\hline 自然膝关节 & 3.015 & 4.852 & 2.864 & 5.275 \\
双动半膝关节 & 5.377 & 4.278 & 2.250 & 3.968 \\
半滕关节 & 5.390 & 6.708 & 3.618 & 7.234 \\
\hline
\end{tabular}

MCCANN 等 ${ }^{[27]}$ 研究了软骨的接触应力对摩擦 因数、摩擦切应力和软骨退化之间的关系, 试验结 果发现, 软骨接触应力的增加直接导致摩擦因数、 摩擦切应力和软骨退化的增加。因此, 半膝关节假 体有可能造成关节面磨损。而双动定制化半膝关节 假体在降低摩擦和磨损的效果明显优于半膝关节假 体; 在某些情况下, 由于置换后关节匹配度的改进, 双动定制化半膝关节假体组内软骨所受接触应力小 于自然关节条件软骨的接触应力, 表明假体置换后 生物力学环境能够获得重建, 甚至提高。

\section{5 体外稳定性试验与临床试验}

体外稳定性试验见图 9, 试验结果见表 8、9。 研究发现, 当膝关节进行深弯曲运动(弯曲角度 $135^{\circ}$, 见表 8$)$, 韧带长度差值最大。根据式(2)计算
得出此时人工韧带拉力为 $390 \mathrm{~N}$; 当胫骨前移 $7.2 \mathrm{~mm}$ (表 9)时, 人工㓞带拉力最大, $510 \mathrm{~N}$ 。由于 试验采用 LARS 人工韧带(纤维支数为 60 根) 可承受 $2500 \mathrm{~N}$ 的抗拉力 ${ }^{[28]}$, 完全可以承受患者运动过程 所造成的对人工韧带的拉力，满足关节的稳定性的 受力要求。

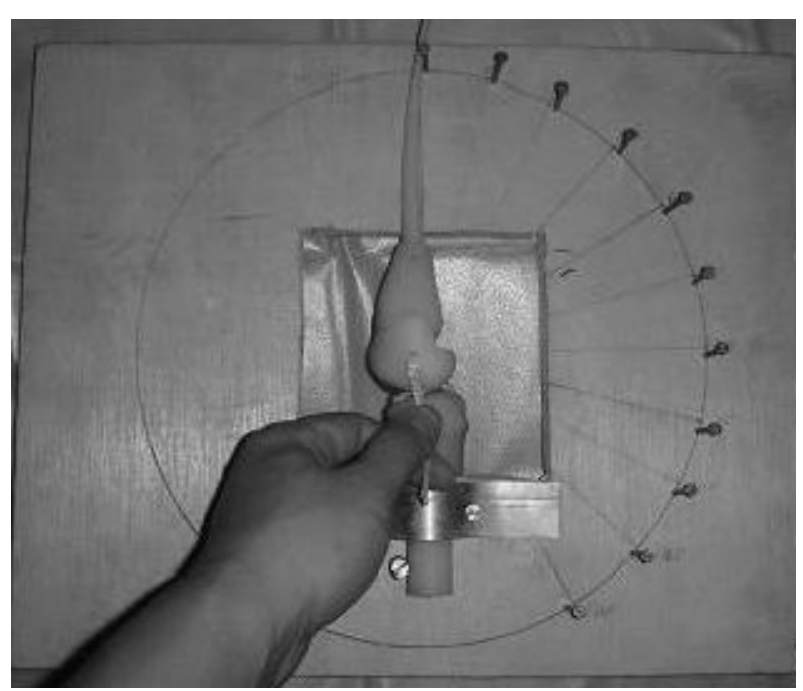

图 9 体外稳定性试验图

表 8 模拟膝关节屈曲状态下韧带长度试验值

\begin{tabular}{|c|c|c|c|c|c|c|c|c|}
\hline \multirow{2}{*}{$\begin{array}{c}\text { 屈曲角度 } \\
\left./^{\circ}\right)\end{array}$} & \multicolumn{8}{|c|}{ 㓞带长度/mm } \\
\hline & 外侧 & 前叉 & 外叉 & 差值 & 内侧 & 后叉 & 内叉 & 差值 \\
\hline 0 & 17.25 & 19.94 & 37.19 & 0 & 16.61 & 11.24 & 27.85 & -10.76 \\
\hline 60 & 17.25 & 19.94 & 37.19 & 0 & 16.61 & 11.24 & 27.85 & -10.76 \\
\hline 75 & 19.39 & 11.32 & 30.71 & -6.48 & 16.58 & 10.78 & 27.36 & -11.25 \\
\hline 90 & 21.64 & 13.07 & 34.71 & -2.48 & 18.29 & 11.11 & 30.40 & -8.21 \\
\hline 105 & 23.65 & 11.62 & 35.27 & -1.92 & 20.36 & 18.25 & 38.61 & 0 \\
\hline 120 & 24.25 & 11.70 & 35.95 & -1.24 & 22.85 & 18.02 & 40.87 & 2.26 \\
\hline 135 & 24.10 & 13.08 & 37.18 & -0.01 & 25.29 & 16.72 & 42.01 & 3.40 \\
\hline
\end{tabular}

表 9 模拟胫骨前后移极限状态下韧带长度试验值

\begin{tabular}{ccccccccc}
\hline \multirow{2}{*}{$\begin{array}{c}\text { 移动状态 } \\
\text { (极限值) }\end{array}$} & \multicolumn{9}{c}{ 㓞带长度 $/ \mathrm{mm}$} \\
\cline { 2 - 8 } 外侧 & 前叉 & 外叉 & 差值 & 内侧 & 后叉 & 内叉 \\
\hline 前移 $(7.2 \mathrm{~mm})$ & 15.71 & 26.84 & 42.55 & 4.36 & 18.83 & 5.07 & 23.90 \\
后移(4.8 mm) & 18.67 & 17.37 & 36.04 & -1.15 & 13.85 & 16.45 & 30.30 & -14.71 \\
基准值 & 17.25 & 19.94 & 37.19 & 0 & 20.36 & 18.25 & 38.61 & 0 \\
\hline
\end{tabular}

根据假体设计图, 委托北京威高亚华人工关节 开发有限公司加工定制化双动半膝关节钛合金假 体。手术在第四军医大学西京医院完成。患者在术 后 $15 \mathrm{~d}$ 后便已出院, 其身体状态恢复良好, 无其他 不适症状。术后假体安装良好, 无脱位现象, 见图 10 。短期随访确定, 患者在出院 1 个月后便可在支 具辅助下行走, 恢复了部分行走能力; 临床上可观 察到患者在完成深度屈曲运动时新型假体的双滑动 过程。但假体的长期疗效仍需要后期随访。

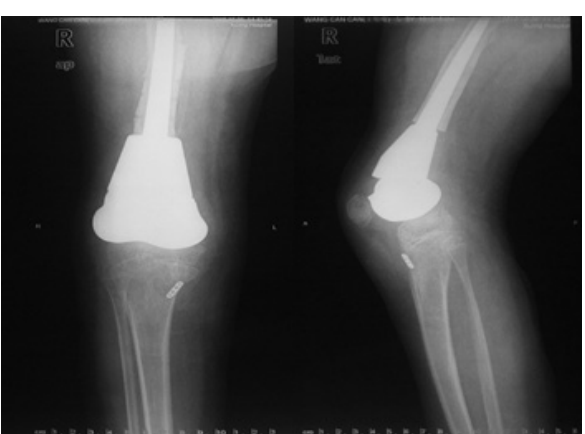

图 10 患者假体置换术后 $\mathrm{X}$ 光片 


\section{4 结论}

（1）提出在半膝关节上构建 “双滑动” 机构与 定制化关节面, 来减少对对侧软骨的摩擦磨损, 增 加假体与周围组织的匹配性和假体运动的稳定性的 设想是可行的。该研究为单侧膝关节损伤患者, 特 别是青少年患者术后骨生长的生物学重建及后期关 节功能的良好恢复提供了有效的治疗方法。

(2) 提出了一套定制化关节设计、模拟分析与 快速评估的整套方法, 从接触力学的角度模拟分析 验证定制化假体在降低关节摩擦和磨损的方法, 以 快速原型零件构建体外稳定性试验平台, 为以后定 制化人工关节的设计与评估提供了进一步的理论分 析基础和手段。

\section{参 考 文 献}

[1] PIERO P. Osteosarcoma (Osteogenic sarcoma)[J]. Orphanet Journal of Rare Diseases, 2007, 2(1): 6 .

[2] FINN H, SIMON M. Limb-salvage surgery in the treatment of osteosarcoma in skeletally immature individuals[J]. Clinical Orthopaedics and Related Research, 1991(262): 108-118.

[3] 袁涛, 许建波, 许哲, 等. 半膝关节假体置换与半膝 骨关节移植保肢治疗膝关节恶性骨肿瘤的 12 例 2 组对 照比较 $[\mathrm{J}]$. 中国组织工程研究与临床康复, 2008, 12(48): 9523-9527.

YUAN Tao, XU Jianbo, XU Zhe, et al. Semi-knee-osteoarticular allograft versus semi-knee-joint prosthesis replacement for malignant tumors: Control study of 12 cases[J]. Journal of Clinical Rehabilitative Tissue Engineering Research, 2008， 12(48): 9523-9527.

[4] MOOH K, KANG J, LEE T, et al. Degeneration of acetabular articular cartilage to bipolar hemiarthroplasty[J]. Yonsei Medical Journal, 2008, 49 (5): 719.

[5] 曾水平, 陈维善, 郭建中, 等. 骨水泥双极式半髋关 节与全髋关节置换治疗高龄股骨颈骨折的比较 $[\mathrm{J}]$. 浙 江医学, 2007, 29(7): 671-672.

ZENG Shuiping, CHEN Weishan, GUO Jianzhong, et al. Comparative research on semi-hip-joint versus total-hip-joint prosthesis replacement for femoral neck fracture[J]. Zhejiang Medical Journal, 2007, 29(7) : 671-672.

[6] 史廷春, 张人佶, 颜永年, 等. 快速成形外耳软骨支架 [J]. 机械科学与技术，2003，22(3)：477-479.
SHI Tingchun, ZHANG Renjie, YAN Yongnian, et al. Rapid manufacturing scaffold of auricular cartilage for macrotia[J]. Zhejiang Medical Journal, 2003, 22(3): 477-479.

[7] HE J, LI D, LU B, et al. Custom fabrication of a composite hemi-knee joint based on rapid prototyping $[\mathrm{J}]$. Rapid Prototyping Journal, 2006, 12: 198-205.

[8] LIN Y, WANG C, DAI K. Reverse engineering in CAD model reconstruction of customized artificial joint[J]. Medical Engineering \& Physics, 2005, 27: 189-193.

[9] SINGER J, LAMONTAGE M. The effect of functional knee brace design and hinge misalignment on lower limb joint mechanics [J]. Clinical Biomechanics, 2008, 23(1): $52-59$.

[10] 邱郡, 张文光, 吴刚, 等. 关节软骨的仿生设计 [J]. 生 物医学工程学报, 2008(1): 182-185.

QIU Jun, ZHANG Wenguang, WU Gang, et al. Bionic design of articular cartilage $[\mathrm{J}]$. Journal of Biomedical Engineering, 2008(1): 182-185.

[11] SINGARE S, LIU Y, LI D, et al. Fabrication of customised maxillo-facial prosthesis using computeraided design and rapid prototyping techniques[J]. Rapid Prototyping Journal, 2006, 12(4): 206-213.

[12] 刘葴，李涤尘，周丽斌，等. 定制化柔性下领骨钛替代 物的有限元优化分析及动物试验 $[\mathrm{J}]$. 机械工程学报, 2010，46(5): 133-138.

LIU Wei, LI Dichen, ZHOU Libin, et al. Optimized finite element analysis of customized flexible mandible titanium substitute and its animal experiment[J]. Journal of Mechanical Engineering, 2010, 46(5): 133-138.

[13] WU Z, LIU Y, SINGARE S, et al. Animal model for evaluation of strain gauge in mandibular distraction osteogenesis in rabbits[J]. British Journal of Oral \& Maxillofacial Surgery, 2007, 45: 633-636.

[14] SINGARE S, LIAN Q, WANG W P, et al. Rapid prototyping assisted surgery planning and custom implant design[J]. Rapid Prototyping Journal, 2009, 15: 19-23.

[15] PAPAIOANNOU G, NIANNIOS G, MITROGIANNIS C, et al. Patient specific knee joint finite element model validation with high accuracy kinematics from biplane dynamic Roentgen stereogrammetric anlysis[J]. Journal of Biomechanics, 2008, 41：2633-2638.

[16] HARRYSSON O L, HOSNI Y A, NAYFEH J F. Custom-designed orthopedic implants evaluated using finite element analysis of patient-specific computed 
tomography data: Femoral-component case study [J]. BMC Musculoskeletal Disorders, 2007, 8: 91.

[17] JUN Y, CHOI K. Design of patient-specific hip implants on the 3D geometry of the human femur [J]. Advances in Engineering Software, 2010, 41(4): 537-547.

[18] HEEVER D J, SCHEFFER C, ERASMUS P, et al. Contact stresses in a patient-specific unicompartmental knee replacement[J]. Clinical Biomechanics, 2011, 26(2): 159-166.

[19] 吕后山. 人工关节外科学[M]. 北京: 科学出版社, 1998. LÜ Houshan. Artificial joint surgery[M]. Beijing: Science Press, 1998.

[20] COMPLETO A, TALAIA P, FONSECA F, et al. Relationship of design features of stemmed tibial knee prosthesis with stress shielding and end-of-stem pain [J]. Materials and Design, 2009, 30: 1391-1397.

[21] 王臻, 滕勇, 李涤尘, 等. 基于快速成型的个体化人 工半膝关节的研制——计算机辅助设计与制造 $[\mathrm{J}]$. 中 国修复重建外科杂志, 2004，18(5)：347-351.

WANG Zhen, TENG Yong, LI Dichen, et al. Fabrication of custom-made artificial semi-knee joint based on rapid prototyping technique: Computer-assisted design and manufacturing $[\mathrm{J}]$. Chinese Journal of Reparative and Reconstructive Surgery, 2004, 18(5): 347-351.

[22] 张涛, 王致, 尹庆水, 等. 复合人工骨半膝关节系统 的仿生制造 $[\mathrm{J}]$. 中国组织工程研究与临床康复, 2009, 13(13): 2415-2418.

ZHANG Tao, WANG Zhen, YIN Qingshui, et al. Biomimetic fabrication of hemi-knee system compounded with artificial bone[J]. Journal of Clinical Rehabilitative Tissue Engineering Research, 2009, 13(13): 2415-2418.

[23] PAWASKAR S S, INGHAM E, FISHER J, et al. Fluid load support and contact mechanics of hemiarthroplasty in the natural hip joint[J]. Medical Engineering \& Physics, 2011, 33(1): 96-105.

[24] 刘鹏, 王㮹, 李涤尘, 等. 定制化铰链内滚动式双动
人工半膝关节假体的设计与研究 $[\mathrm{J}]$. 科学技术与工程, 2009, 9(7): 1690-1695.

LIU Peng, WANG Zhen, LI Dichen, et al. Design and Investigation of Hinge with Inter-rolling double-action customized semi-knee artificial prosthesis[J]. Science Technology and Engineering, 2009, 9(7): 1690-1695.

[25] MANDELL J A, CARTER D R, GOODMAN S B, et al. A conical-collared intramedullary stem can improve stress transfer and limit micromotion[J]. Clin. Biomech., 2004. 19(7): 695-703.

[26] 王尉年. 人工膝关节——理论基础与临床应用 $[\mathrm{M}]$. 上 海: 复旦大学出版社, 2004 .

WANG Weinian. Artifical knee prosthesis: Theoretical basis and clinic application[M]. Shanghai : Fudan University Press, 2004.

[27] MCCANN L, INGHAM E, JIN Z, et al. An investigation of the effect of conformity of knee hemiarthroplasty designs on contact stress, friction and degeneration of articular cartilage: A tribological study[J]. Journal of Biomechanics, 2009, 42 (9): 1326-1331.

[28] 康一凡，李明，林志金. LARS 人工韧带重建膝交叉韧 带实用手术技巧 $[\mathrm{M}]$. 上海: 第二军医大学出版社, 2010 .

KANG Yifan, LI Ming, LIN Zhijin. LARS artifical ligament reconstruction of the practical surgical skills[M]. Shanghai: Second Military Medical University Press, 2010.

作者简介: 靳忠民, 男, 1963 年出生, 博士, 教授, 博士研究生导师。 主要研究方向为人工关节与生物摩擦学。

E-mail: zmjin@mail.xjtu.edu.cn 连芩(通信作者), 女, 1971 年出生, 博士, 副教授, 硕士研究生导师。 主要研究方向为快速制造与生物制造。

E-mail: lqiamt@mail.xjtu.edu.cn

李涤尘, 男, 1964 年出生, 博士, 教授, 博士研究生导师。主要研究方 向为增材制造与生物制造。

E-mail: dcli@mail.xjtu.edu.cn 